\title{
Identification of the membrane-spanning domain of glycoprotein 45 in bovine immunodeficiency virus
}

\author{
W. SHEN, J. FENG, Z. LIU, D. DIAO, CH. LIU, X. KONG* \\ Laboratory of Medical Molecular Virology, School of Medicine, Nankai University, Weijin Road No.94, Nankai District, \\ Tianjin, P. R. China
}

Received March 20, 2017; revised December 18, 2017; accepted July 10, 2018

\begin{abstract}
Summary. - The membrane-spanning domain (MSD) of the transmembrane subunit (TM) anchors the envelope glycoprotein (Env) on the lipid bilayer of the host cell membrane and virions. Its functions include membrane fusion efficiency and intracellular trafficking of the lentivirus envelope protein. Our study aimed to determine the MSD of bovine immunodeficiency virus (BIV) glycoprotein 45 (gp45) and reveal structural characteristics of the BIV Env protein. We have predicted the region of the BIV MSD and obtained the sequence using bioinformatics software. Various kinds of assays, including analogy analysis, fluorescence microscopy, and dye-transfer-based assays, were carried out to validate the prediction. The results, for the first time, show that the BIV MSD is located at the D170 to M191 amino acids of gp45, and the identified MSD divides gp45 into the extracellular domain (ED), MSD and cytoplasmic domain (CT). We further found that the BIV MSD had a similar structure and function as the HIV MSD using amino acid sequence alignment and fluorescence microscopy. Additionally, the dye-transfer-based assay demonstrates that deletion of the BIV MSD efficiently decreases cell-cell fusion. Based on the identification of the MSD, a "snorkeling" model, in which the flanking charged amino acid residues are buried in the lipid bilayer while their side chains interact with polar head groups, was proposed for the BIV MSD. Ultimately, we further improved the primary structure of the BIV envelope glycoprotein.
\end{abstract}

Keywords: bovine immunodeficiency virus; membrane-spanning domain; bioinformatics prediction; cellcell fusion; primary structure; "snorkeling" model

\section{Introduction}

BIV belongs to the lentivirus genus of the subfamily Orthoretrovirinae of the Retroviridae family and causes a persistent viral infection in cattle and buffalo. Infection with BIV has been associated with lymphadenopathy, lymphocytosis, central nervous system lesions, progressive weakness, a decreased lymphocytic blastogenic response

"Corresponding author. E-mail: kongxh@nankai.edu.cn; phone: +86-022-23504347.

Abbreviations: $\mathrm{BIV}=$ bovine immunodeficiency virus; $\mathrm{CT}=\mathrm{cy}-$ toplasmic domain; $\mathrm{ED}=$ extracellular domain; Env = envelope; HIV = human immunodeficiency virus; $\mathrm{MSD}=$ membrane-spanning domain abbreviated; SIV = simian immunodeficiency virus; $\mathrm{SU}=$ surface subunit; $\mathrm{TM}=$ transmembrane subunit and bovine paraplegic syndrome, leading to decreased milk yield and a threat to the survival of the cattle (Bhatia et al., 2013). Further research on BIV is needed to elucidate its possible role in animal health, as well as to understand the molecular mechanisms adopted by related lentiviruses. BIV can significantly reduce the loss of dairy farmers, so its study is important for the control of the disease.

The BIV genome, similar to human immunodeficiency virus (HIV) and other lentiviruses, contains the structural genes gag, pol, env and several accessory genes tat, rev, vif, $v p w, v p y$, and $\operatorname{tm} x$. However, although some studies on BIV are focused on tat (Guo et al., 2013), vif (Zhang et al., 2014) rev (Gomez Corredor and Archambault, 2009; Gomez Corredor and Archambault, 2012) and others (Albernaz et al., 2015; Liu et al., 2015), very scarce attention has been paid to env. The Env of BIV is composed of two subunits: 
gp100, a surface subunit (SU) that binds host cell receptors, and gp45, a transmembrane subunit (TM) that ultimately inserts into the host cell membrane and promotes the fusion. The conservative and variable regions of BIV SU have been defined by the Suarez group (Suarez and Whetstone, 1995). However, the BIV TM functions has not yet been clarified.

In the genus lentivirus of the retroviruses, the TM commonly consists of an extracellular domain (ED), MSD, and cytoplasmic domain (CT) (Weiss, 2003). The extracellular domain mediates membrane fusion. It contains a fusion peptide (Bosch et al., 1989) and two heptad repeat regions, HR1 and HR2 (Chan et al., 1997), which were determined in 2005 (Li et al., 2005). The cytoplasmic domain is important for intracellular trafficking, and it affects the incorporation of Env into the budding viral particle (Salzwedel et al., 1999; Lu et al., 2008; Bhakta et al., 2011; Postler and Desrosiers, 2012). The MSD usually consists of 20-25 highly conserved hydrophobic amino acids and forms an alpha-helix in the lipid bilayer. Several studies have indicated that the MSD plays a particularly important role in membrane fusion. For example, a previous study showed that substituting a leucine residue for the conserved arginine residue within the MSD resulted in a replication-incompetent virus (Owens et al., 1994). Furthermore, fusion activity was severely impaired in MSD-replacement mutants (Miyauchi et al., 2005). Shang et al. have reported that substitutions within the MSD "core" delays the kinetics and reduces the efficiency of cell-cell fusion mediated by HIV Env (Shang and Hunter, 2010).

The MSD of HIV and simian immunodeficiency virus (SIV) have two alternative models. The original HIV-1 MSD model predicts a structure of 25 amino acid residues from K681 to R705 in the form of the alpha helix. The charged amino acid R694 is buried in the membrane. The original SIV MSD is from lysine 164 to lysine 191 and contains an arginine in the membrane at position 180. An alternative "snorkeling" model was first proposed for the SIV MSD (West et al., 2001), and it was also applicable to the HIV-1 MSD (Yue et al., 2009). The "snorkeling" model involves fewer amino acids than the original model. In "snorkeling" model, the MSD forms the alpha-helix. This model has an alpha-helical "core" region, which consists of 12 hydrophobic residues between K681 and R694 in HIV-1 and 15 hydrophobic residues between K689 and R705 in SIV, respectively. This "core" region could be stably accommodated within the membrane. The lysine and arginine residues are buried in the lipid bilayer, while their long side chains are proposed to extend outward to the membrane surface and present the positively charged amino groups to the negatively charged head groups of the lipid bilayers.

Although the MSD is crucial for anchoring Env in the membrane and for virus entry, the structure and function of the BIV MSD are not clear. Therefore, identification of the BIV MSD will reveal structural characteristics of the Env protein and provide the fundamental primary structure for further studies of the BIV Env.

In this study, the MSD regions of the BIV Env were determined using bioinformatics assays and functional verification. For comparison, some work was also performed using the HIV Env protein. Our results show that the BIV MSD is from D170 to M191 amino acids of gp45, as predicted. The amino acid sequence alignment analysis and fluorescence microscopy assay demonstrated that the MSD of BIV and HIV had a similar primary and secondary structure. Furthermore, the fluorescence microscopy assay demonstrated that the MSD of BIV and HIV had common functional characteristics. The dye-transfer-based assay results suggest that the BIV MSD is critical for membrane fusion. Based on the identification of MSD, a "snorkeling" model was proposed for the BIV MSD. This study regarding the BIV Env protein improves knowledge of the Env of lentiviruses, regarding their primary structure and membrane fusion. Moreover, it will facilitate the development of BIV vaccines.

\section{Materials and Methods}

Sequences acquirement and prediction. The env gene of BIVR29 was obtained from NCBI (NC_001413). The membrane-spanning domain of gp45 in BIV was predicted using the TMpred program (http://www.ch.embnet.org/software/TMPRED_form.html), HMMTOP program (http://www.enzim.hu/hmmtop/) (Tusnady and Simon, 1998; Tusnady and Simon, 2001) and TMHMM program (http://www.cbs.dtu.dk/services/TMHMM-2.0/). The Helical Wheel Projections (http://rzlab.ucr.edu/scripts/wheel/wheel.cgi) was used for predicting the secondary structure of MSD. The TMpred program makes a prediction of membrane-spanning regions and their orientation. The prediction is made using a combination of several weight-matrices for scoring. HMMTOP is an automatic server for predicting transmembrane helices and the topology of proteins at the Institute of Enzymology. The method used by this prediction server is described previously (Tusnady and Simon, 2001). Moller et al. showed that TMHMM is currently the best performing transmembrane prediction program (Moller et al., 2001).

Plasmids. BIV127 (Braun et al., 1988), a BIV provirus clone, and HIV pNL4-3 were provided by Dr. Charles Wood (University of Nebraska Lincoln). pcBIV, where the Env fragment of BIV, was inserted into the pcDNA3.1 (+) vector, was provided by Dr. Wentao Qiao (Nankai University). The pEGFP-C1 vector (Clontech Laboratories, Inc., USA) carries the enhanced green fluorescent protein (EGFP) gene as a reporter gene and the kanamycin-resistance gene as a selection marker.

Plasmids construction. Six plasmids were constructed in our study, and all of them expressed the green fluorescent protein and the objective gene. The objective MSD/NC fragment was synthesized with oligonucleotides by Sangon Biotech (Shanghai, China) with additional EcoRI and BamHI restriction sites. HIV-gp45/BIV- 
gp41 was amplified by PCR from the infectious molecular clone BIV127/pNL4-3. XhoI and EcoRI restriction sequences were added to the $5^{\prime}$ end of the forward and reverse primers, respectively. PCR was carried out at $95^{\circ} \mathrm{C}$ for $2 \mathrm{~min}$, followed by 25 cycles at $95^{\circ} \mathrm{C}$ for $30 \mathrm{~s}, 55^{\circ} \mathrm{C}$ for $30 \mathrm{~s}$, and $72^{\circ} \mathrm{C}$ for $2 \mathrm{~min}$, followed by $1 \mathrm{cycle}$ at $72^{\circ} \mathrm{C}$ for $10 \mathrm{~min}$. The amplified products were subcloned into the pEGFP-C1 vector. In the ligation reaction, $100 \mathrm{ng}$ of vector were used in $20 \mu$ reaction mixture. MSD/NC fragment was in 5:1 molar ratio over vector, while HIV-gp45/BIV-gp41 fragment was in 3:1 molar ratio over vector. The primers used in this study are shown in the Table1.

The plasmids of pcBIV-nonMSD were constructed in this study using Thermo Scientific Phusion Hot Start II High-Fidelity DNA polymerase (Thermo Scientific, USA). PCR was carried out at $98^{\circ} \mathrm{C}$ for $30 \mathrm{~s}$, followed by 25 cycles at $98^{\circ} \mathrm{C}$ for $10 \mathrm{~s}, 55^{\circ} \mathrm{C}$ for $30 \mathrm{~s}$, and $72^{\circ} \mathrm{C}$ for $10 \mathrm{~min}$, followed by $1 \mathrm{cycle}$ at $72^{\circ} \mathrm{C}$ for $10 \mathrm{~min}$. All mutations were confirmed by DNA sequencing using a primer approximately $100 \mathrm{bp}$ upstream from the MSD sequence.

Cell culture. COS-1, BHK21, HeLa and CV-1 cell lines have been used in our study (ATCC, USA). All the cell lines were cultured in Dulbecco's Modified Eagle Medium (DMEM, Gibco, USA) and supplemented with $10 \%$ fetal bovine serum (FBS), $0.1 \%$ penicillin, and $0.1 \%$ streptomycin at $37^{\circ} \mathrm{C}$ and $5 \% \mathrm{CO}_{2}$.

Dye-transfer-based assay. The assay was based on calculation of dye-transfer events between fluorescently labeled target and effector cells, as described previously (Cohen and Melikyan, 1998; Munoz-Barroso et al., 1998). COS-1 cells expressing Env (designated the 'effector cells') were labeled with the cytoplasmic marker CMAC (Molecular Probes, USA). BHK21 cells (the 'target cells') were co-labeled with the cytoplasmic marker Cal-AM (Molecular Probes) and DiI (Molecular Probes). Briefly, a confluent monolayer of COS- 1 cells on a poly-L-lysine (Sigma)-coated slide $\left(1 \times 10^{5}\right.$ cells) was washed twice with PBS and incubated for $30 \mathrm{~min}$ at $37^{\circ} \mathrm{C}$ with DMEM containing $30 \mathrm{mmol} / \mathrm{l} \mathrm{CMAC}$. The BHK21 cells $\left(3 \times 10^{5}\right.$ cells) were co-labeled with $5 \mathrm{mmol} / \mathrm{l} \mathrm{Cal-AM}$ and $1 \mathrm{mmol} / \mathrm{l} \mathrm{DiI}$ in DMEM. To induce fusion, fluorescently labeled effector cells and target cells were then co-cultured at $37^{\circ} \mathrm{C}$ for $3 \mathrm{~h}$. The fusion events were assessed by fluorescence microscopy (Olympus,
Japan). For each sample, fusion events from at least three random different vision fields were collected, and the data were analyzed by overlaying the images using Image-Pro Plus software (Media Cybernetics, USA). The extent of fusion was calculated as the ratio of fused cells (positive for all three dyes) to the sum of fused cells and the number of effector-target cell pairs that did not fuse (Abrahamyan et al., 2003).

Fluorescence microscopy assay for distribution. To determine the intracellular distribution of the envelope proteins, we generated a series of expression vectors (Fig. $2 \mathrm{~d}$ ). HeLa and CV-1 cells were transfected with those expression vectors. After $24 \mathrm{~h}$ of culture, the cells were observed, and images were obtained using fluorescence microscopy (IX71, Olympus, Japan).

\section{Results}

\section{Prediction of membrane-spanning domain}

Three different bioinformatic softwares, TMpred, HMMTOP and TMHMM were applied to predict the MSD of the BIV Env protein. First, BIV gp45 protein sequences were inputted to the prediction programs, respectively. Next, we followed the directions to analyze the sequences. TMpred and HMMTOP predicted the same three domains, however, TMHMM only predicted two domains in BIV gp45 (Fig. 1). We concluded that based on the consistent results predicted by these three programs, 2-27 amino acids and 170-191 amino acids (557-572 and 725-746 amino acids of BIV Env) were candidates for the MSD of gp45 (Fig. 1a, b and c).

\section{Identification of membrane-spanning domain}

BIV, HIV-1 and SIV all belong to the lentivirus family (ICTV 2011), and they have similar morphological, immunological and genetic characteristics (Gonda et al., 1987). Particularly, the products of the accessory genes ' $v$ if and 'tat', as well as those of the structural genes ' $g a g$ ', 'pol' and

Table 1. Primers used in this study

\begin{tabular}{|c|c|}
\hline Primers & Sequence $\left(5^{\prime}-3^{\prime}\right)$ \\
\hline BIV-MSD-F & AATTCCATGGACTGGATAAAAATCATTATAGTAATCATTGTACTTTGGCTTCTGATAAAGATTCTCCTAGGTATGG \\
\hline BIV-MSD-R & GATCCCATACCTAGGAGAATCTTTATCAGAAGCCAAAGTACAATGATTACTATAATGATTTTTATCCAGTCCATGG \\
\hline HIV-MSD-F & AATTCCATGAAATTATTCATAATGATAGTAGGAGGCTTGGTAGGTTTAAGAATAGTTTTTGCT \\
\hline HIV-MSD-R & GATCCCCTAACTCTATTCACTATAGAAAGTACAGCAAAAACTATTCTTAAACCTACCAAG \\
\hline BIV-gp45-F & CCGCTCGAGCGGCGATGGCAGTCGGACTAGCAATATTC \\
\hline BIV-gp45-R & 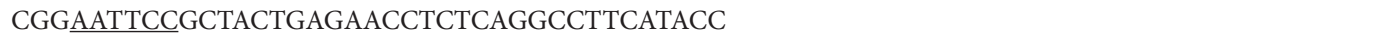 \\
\hline HIV-gp41-F & CCGCTCGAGCGGCGATGGCAGTGGGAATAGGAGCTTTG \\
\hline HIV-gp41-R & CGGAATTCCGTTATAGCAAAATCCTTTCCAAGCCC \\
\hline BIV-NC-F & AATTCCATGCTGTCCATGGGAGTAGGAATGCTACTAGGAGTATATTGTCAGTTACCAGACACACTGATTTGGG \\
\hline BIV-NC-R & GATCCCCAAATCAGTGTGTCTGGTAACTGACAATATACTCCTAGTAGCATTCCTACTCCCATGGACAGCATGG \\
\hline
\end{tabular}


(a)

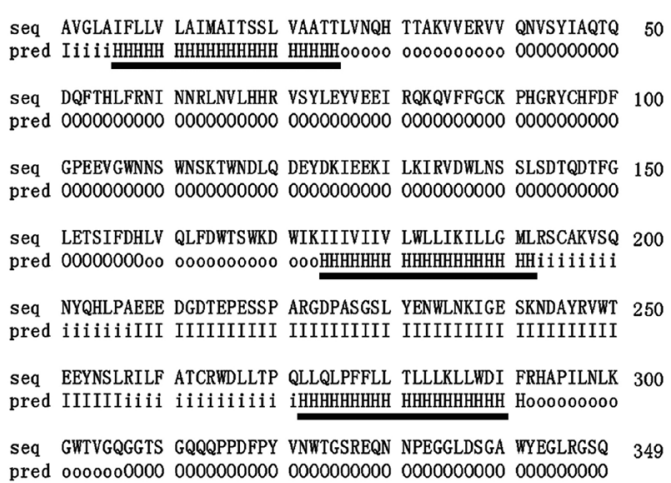

Seq AVGLAIFLLV LATMAITSSL VAATTLVNQH TTAKVVERVV QNVSYIAQTQ 50 pred III i i HHHHH HHHHHHHHH HHHHHO0000 00000000000000000000 SEq DQFTHLFRNI NNRLNVLHHR VSYLEYVEET RQKQVFFGCK PHGRYCHFDF 100 pred 0000000000000000000000000000000000000000000000000 seq EEYNSLRILF ATCRYDLLTP OLLOLPFFLL TLLLKLLWDI FRHAPILNLK 300 seq GWTVGQGGTS GQQQPPDFPY VNWTGSREQN NPEGGLDSGA WYEGLRGSQ 349 pred 0000000000000000000000000000000000000000000000000

(c)

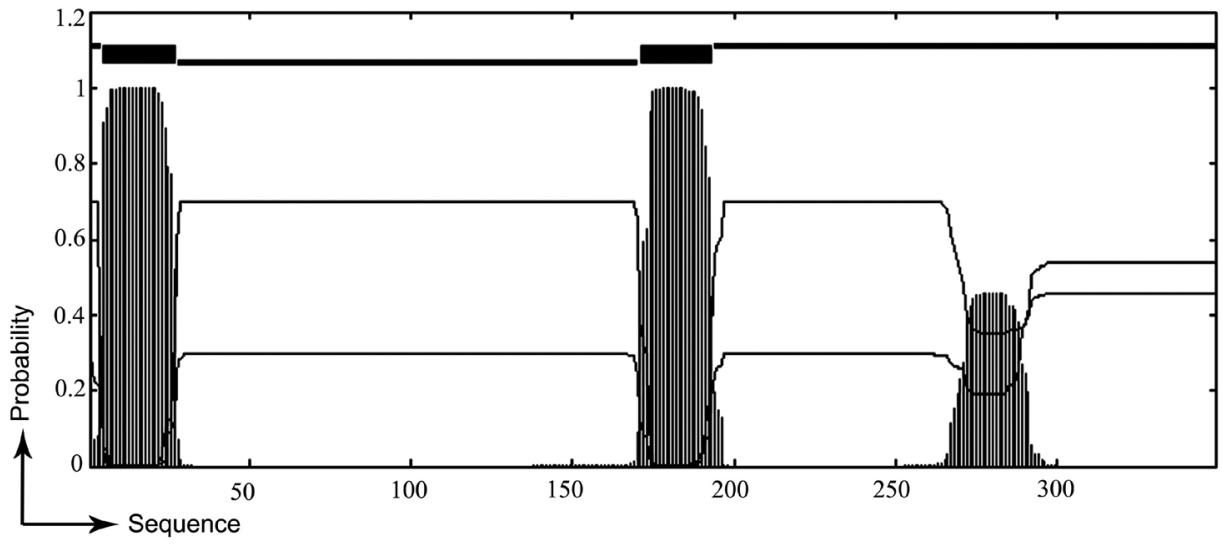

Fig. 1

Prediction of the BIV MSD by using the program HMMTOP, TMpred and TMHMM

(a) The result of the BIV MSD prediction using the HMMTOP program. Three regions, 6-25, 174-192, and 272-291, are BIV-MSD candidates. Shorthand: inside loop (I), inside tail (i), membrane helix (h), outside tail (o) and outside loop (O). (b) The result of the BIV-MSD prediction using the TMpred program. Only scores above 500 are considered significant. Three regions, 2-21, 170-189, and 267-287, were predicted. (c) The result of the BIV-MSD prediction using the TMHMM program. TMHMM predicted the MSD probably in the 2-27 and 170-191 of the BIV gp45.

'env', of BIV have some sequence similarities to their counterparts in HIV-1. Therefore, to identify the MSD of BIV, we can use the protein primary structure of HIV for reference. The Env of HIV-1 is synthesized as a precursor of gp160 and then processed into a heterodimer consisting of gp 120 and gp41. The gp41 subunit is organized into three major domains: an extracellular domain, a MSD and a cytoplasmic domain (Fig. 2a).

The extracellular domain contains the major fusion determinants: N-terminal hydrophobic region known as the fusion peptide (Bosch et al., 1989; Freed et al., 1990; Freed et al., 1992) and an alpha-helical coiled-coil structures referred to as the heptad repeat regions HR1 and HR2 (Lu et al., 1995; Chan et al., 1997; Tan et al., 1997; Weissenhorn et al., 1997) (Fig. 2a). The MSD is located downstream of HR1 and HR2. Li et al. predicted the region of BIV HR1

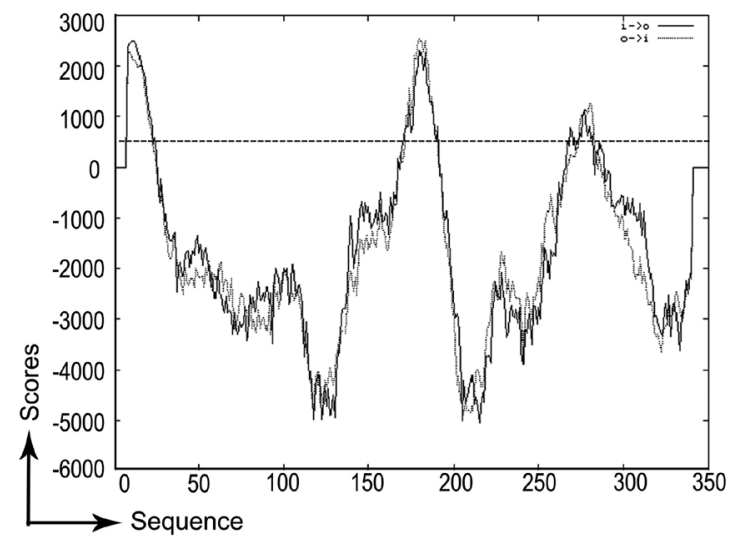


(a)
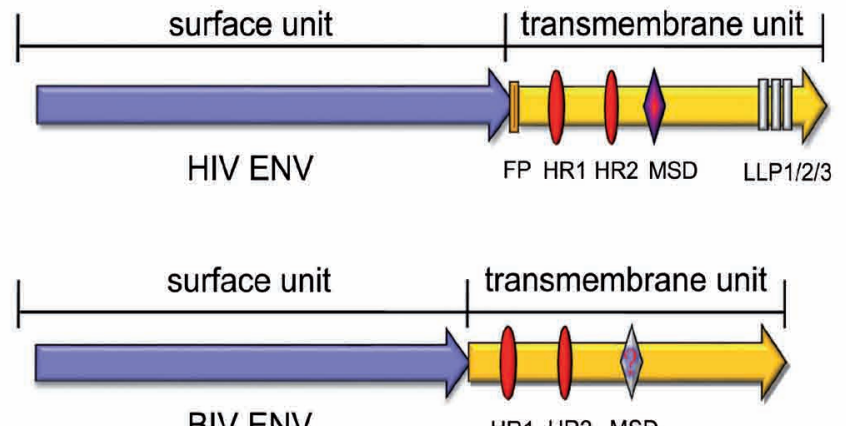

BIV ENV

(b)

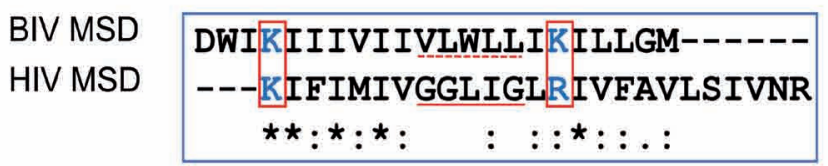

(d)

\begin{tabular}{|c|c|c|c|c|}
\hline pBIVgp45/HIVgp41 & EGFP & ER & MSD & CD \\
\hline pBIV-NC & EGFP & NC & & \\
\hline pBIV/HIV-MSD & EGFP & MSD & & \\
\hline
\end{tabular}

Fig. 2 (c)

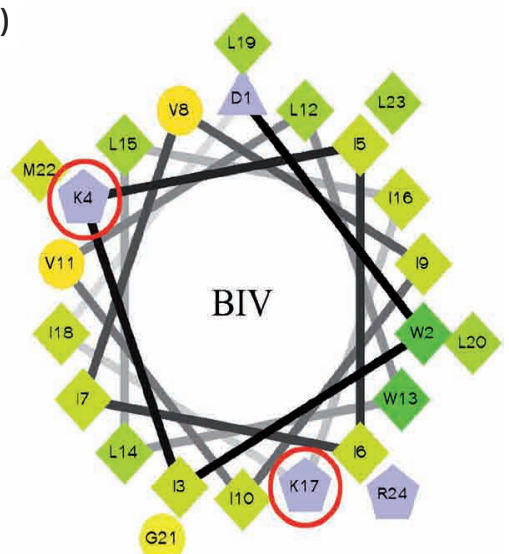

G21

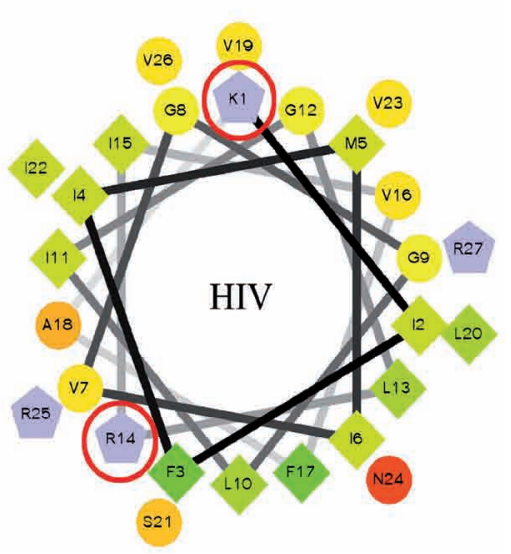

Identification of the BIV MSD

(a) Schematic diagram of BIV and HIV Env. (b) The comparison of the homology of the BIV and HIV MSD. Asterisk $\left(^{*}\right)$ indicates a single, fully conserved amino acid residue; colon (:) indicates conservation between groups of strongly similar properties; period (.) indicates conservation between groups of weakly similar properties. (c) Helix wheel analysis of the predicted coiled-coil regions of BIV and HIV MSD. The output presents the hydrophilic residues as circles, hydrophobic residues as diamonds, potentially negatively charged as triangles, and potentially positively charged as pentagons. The most hydrophobic residue is green, and the amount of green decreases proportionally to the hydrophobicity, with zero hydrophobicity coded as yellow. Hydrophilic residues are coded red, with pure red being the most hydrophilic (uncharged) residue, and the amount of red decreasing proportionally to the hydrophilicity. The potentially charged residues are light blue. (d) Schematic diagram of the constructed plasmids of BIV and HIV used in this study.

HIV MSD, as a comparison, underwent the same analysis. The results showed that BIV MSD can form an alpha-helix and the secondary structure of BIV MSD is similar to HIV MSD. They are composed of hydrophobic amino acids and the basic amino residues are positioned on opposite sides of the MSD alpha-helix assumption.

The BIV MSD and HIV MSD changed the intracellular distribution of GFP according to their hydrophobic properties

To further verify the prediction of the BIV MSD, we constructed a series of GFP-tag fusion protein expressing plasmids (Fig. 2d), previously described in the materials and methods. HeLa and CV-1 cells were transfected with the pEGFP-C1 vectors pBIVgp45 and pHIVgp41, respectively.
After $24 \mathrm{~h}$ of cultivation, images of the cells were taken using fluorescence microscopy. The results showed that BIV gp45 and HIV gp41 changed the intracellular distribution of GFP. We speculated that the MSD of TM (gp45/gp41) plays a very important role in the intracellular distribution of the fluorescent protein. To verify this hypothesis, we examined the distribution of pBIV-MSD in HeLa cells and CV-1 cells. We observed that pBIV-MSD and pBIVgp45 had the same fluorescence distribution, with GFP distributed throughout the cytoplasm (Fig. 3). The same result was obtained in HIV. These results suggest that the predicted BIV MSD has the same properties to change GFP distribution as HIV MSD. We think that the hydrophobic properties of the MSD are the reason for BIVgp45 distribution in the cytoplasm. We inserted the 52-74 amino acids of BIV gp45 into the 
(a)

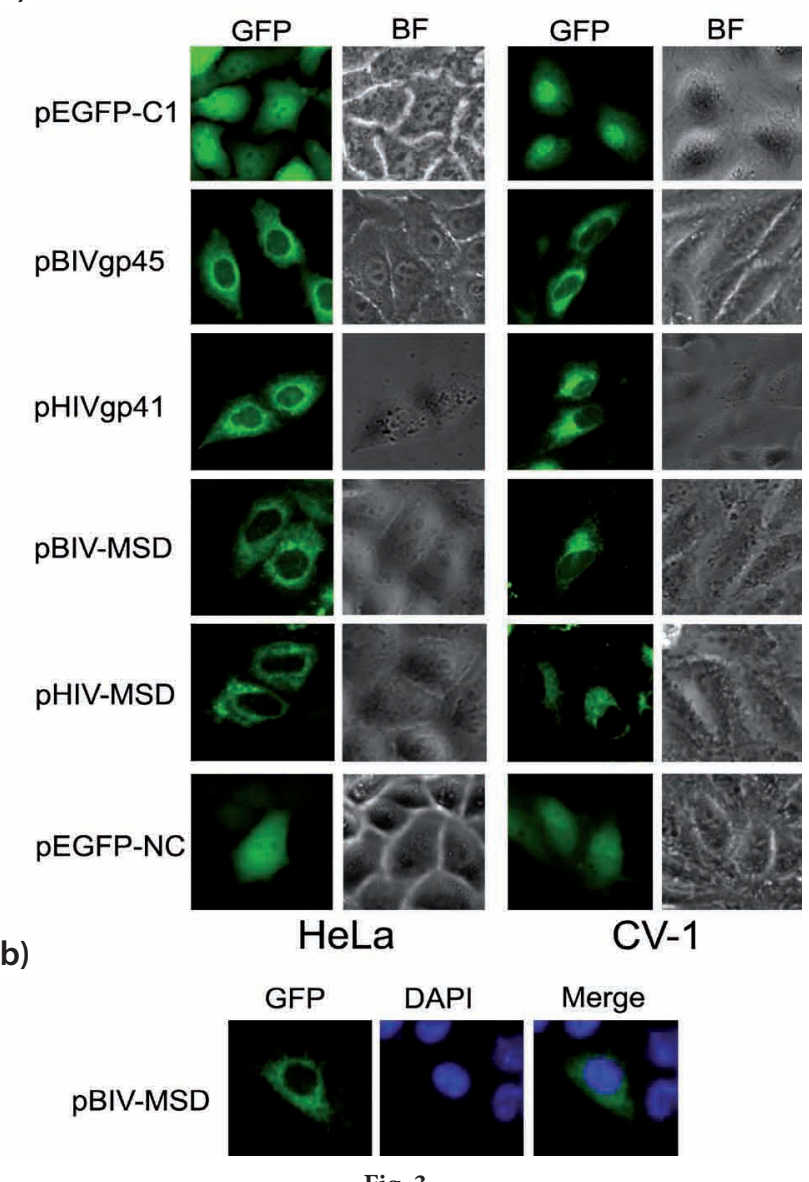

The localization analysis of the BIVgp45 and HIVgp41 fragments (a) HeLa or CV-1 cells were transfected with 6 different plasmids, including pEGFP-C1, pBIVgp45, pHIVgp41, pBIV-MSD, pHIV-MSD, and pEGFPNC. After 24 h of cultivation, the cells were observed, and images were obtained using fluorescence microscopy. (b) HeLa cells were transfected with pBIV-MSD for 24h. DAPI was used to stain the cell nuclei (blue). Images were obtained using fluorescence microscopy.

pEGFP-C1 vector as a control and designated it as pBIV-NC (Fig. 2d). The results showed that the 52-74 amino acids of BIV gp45 could not change the intracellular distribution of GFP (Fig. 3). These data suggest that the intracellular distribution of BIVgp45 is probably due to the highly hydrophobic amino acid sequence of the MSD.

\section{Cell-cell fusion efficiency was significantly decreased by} the deletion of the BIV MSD

The previous data suggest the involvement of the HIV MSD in membrane fusion (Miyauchi et al., 2005; Shang et al., 2008; Kondo et al., 2010; Bhakta et al., 2011; Reuven et al., 2012), and the length of this region is more important than its amino acid conservation (Yue et al., 2009).
Therefore, we further assumed that the BIV MSD should be involved in membrane fusion too. To determine the ability of BIV MSD to mediate cell-cell fusion, the dyetransfer-based assay was performed. Briefly, COS-1 cells were transfected with pcBIV or pcBIV-nonMSD, and cocultured with BHK21 cells for $3 \mathrm{~h}$, and then underwent the cell-cell fusion assay and fluorescent probe transfer assay. The COS- 1 cells as effector cells were loaded with 7-amino4-chloromethylcoumarin (CMAC; blue), while the BHK21 target cells were loaded with the lipophilic octadecyl (C18) indocarbocyanine probe DiI (red) and the cell-permeant and non-fluorescent compound Calcein-AM (green). The fused cells containing all three dyes were then quantified using fluorescence microscopy (Fig. 4a). The statistical results suggested that the Env proteins containing the MSD regions exhibited higher fusion efficiency than those with an MSD deletion (Fig. 4b).

\section{The "snorkeling" model proposed for the BIV MSD}

The MSD of HIV and SIV have two alternative models, original model and "snorkeling" model (West et al., 2001; Yue et al., 2009). In our study, we found that the original model cannot be applicable for BIV MSD, because the BIV MSD has less than 25 amino acids. So, we have proposed "snorkeling" model for BIV MSD. According to previous reports (West et al., 2001; Yue et al., 2009), the "snorkelling" model needs to fulfil the characteristics as: the MSD forms alpha helix in the lipid bilayer and contains an alpha-helical "core" region; the "core" region and two basic amino acids are buried in the lipid bilayer; the total lengths of "core" region and basic amino acid side chains should be between 30-40 $\AA$; side chains of basic amino acids are proposed to extend outward to the membrane surface and present the positively charged amino groups to the negatively charged head groups of the lipid bilayers.

As shown in Fig. 5a, compared with the determined HIV MSD, the BIV MSD is from D725 to M746 (D170 to M191 of gp45) and contains two lysins in the membrane at position 728 and 741 . This model predicts a hydrophobic intramembrane core of 12 amino acid residues (same to 12 amino acid residues in the HIV MSD) between K728 and K741. The length of alpha-helix formed from "core" region and two basic amino acids is approximately $21 \AA$ and the length of the side chain of lysine is approximately $6.4 \AA$. Thus, the addition of the length of the 14-amino-acid alpha-helix and those of the two side chains of K728 and K741 reaches $33.8 \AA$. The two lysins of BIV (compared to the lysine and arginine residues in the HIV MSD) are buried in the lipid bilayers, while their long side chains are proposed to extend outward to the membrane surface and present the positively charged amino groups to the negatively charged head groups of the lipid bilayers. This result suggests that the length of the BIV 
(a),
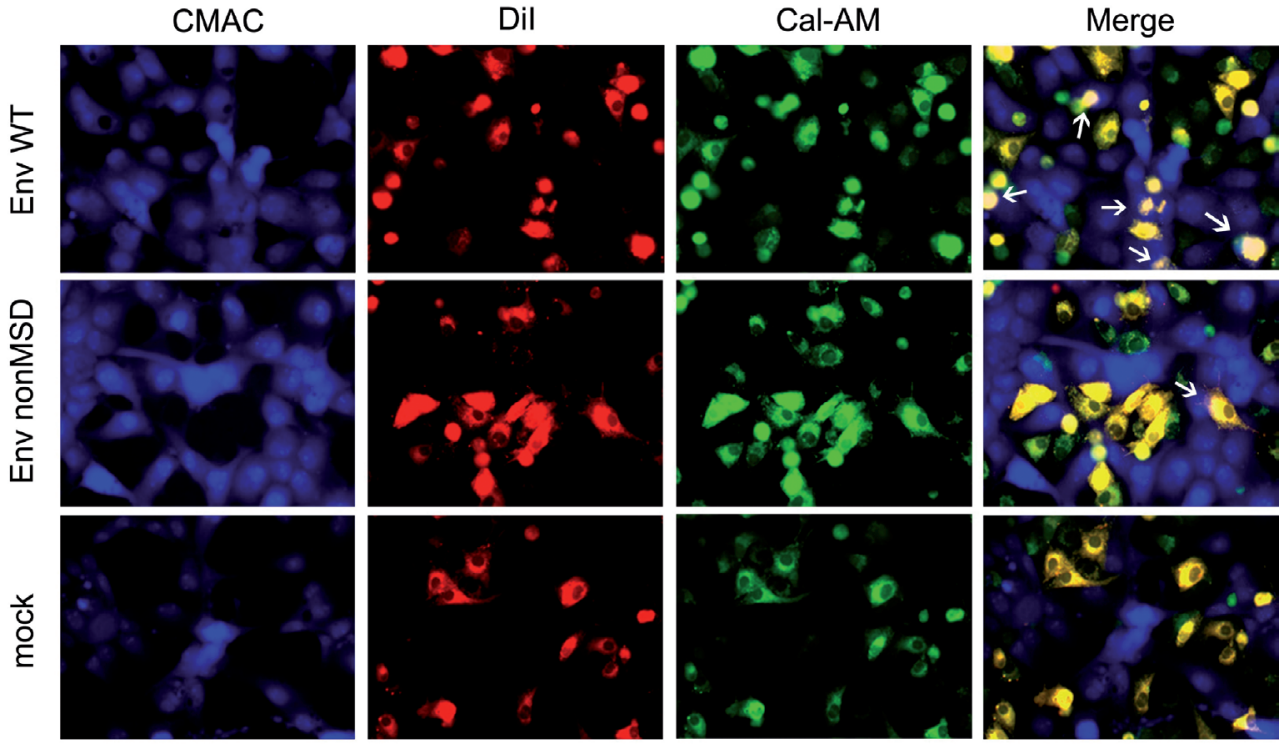

(b)।

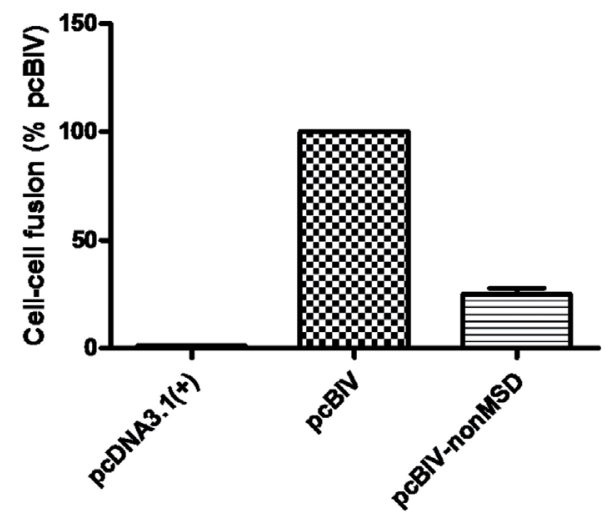

Fig. 4

Fusion assays by dye-transfer-based assay

(a) Fluorescent microscopic images of cell-cell fusion mediated by Env using a dye-transfer-based assay. Effector (COS-1) cells expressing Env were labelled with CMAC (blue). Target (BHK21) cells were co-labelled with DiI (red) and Calcein-AM (green). Fluorescent images for each dye were acquired. Fused cells are depicted by arrowheads. (b) Comparison of the fusion efficiency of Env-WT and Env-nonMSD. The results are shown as the means $\pm \mathrm{SD}$ of three independent experiments.

MSD is great enough to cross the viral membrane, and the "snorkeling" model is more suitable for the MSD structure model than the original model.

\section{Discussion}

Env plays an essential role in the viral replication cycle by mediating the fusion between viral and cellular membranes during viral entry. The MSD usually consists of 20-25 highly conserved hydrophobic amino acids and forms an alpha-helix in the lipid bilayer. The MSD not only anchors Env on the lipid bilayers, but it is also associated with Envmediated membrane fusion (Raja et al., 1994; Wilk et al., 1996; Miyauchi et al., 2005; Welman et al., 2007). Prior research regarding the MSD mainly focused on HIV and

SIV. However, these studies have not considered BIV. In this study, we aimed to identify the MSD of gp45 in BIV using bioinformatics predictions and experimental demonstrations and to determine the primary structure of BIV Env for use in further studies.

Here we have determined that the MSD spans from D170 to M191 amino acid in the BIV gp45, including 18 hydrophobic amino acids. In the secondary structure, basic amino acids are located on both sides of the alpha-helix, which is similar to other lentiviruses. In addition, a series of experiments verified the prediction. These studies also demonstrated that all the envelope proteins from different lentiviruses have common structural features. We further demonstrated that the MSD deletion mutant inhibited cellcell fusion using dye-transfer-based fusion analysis. The results proved that MSD is required for fusion. 
It has been reported that a length of 21 amino acid residues was sufficient for SIV Env to be incorporated into viral particles (West et al., 2001). Therefore, the entire 22-aminoacid region of BIV is enough to mediate viral incorporation and infectivity. While some transmembrane proteins do contain charged amino acid residues in their MSD, it is normally considered to be energetically unfavorable without a mechanism to neutralize the charge (Cosson and Bonifacino, 1992; Fujiyoshi, 1998). In BIV, the MSD is 22 amino acids long, including 18 hydrophobic amino acids and 2 basic amino acids. The MSD of HIV contains well-conserved glycine residues that form the GXXXG motif (G, glycine; X, other amino acid residues) (Senes et al., 2004), a motif often found at the helix-helix interface of membrane-spanning alpha-helices. The primary structure of the MSD of HIV-1 Env also has two basic amino residues in its hydrophobic amino acid sequence. Interestingly, we found that the BIV MSD does not contain the GXXXG motif (Miyauchi et al., 2005). We consider that this may be a reason for the lower spread of BIV in comparison to HIV. Downstream of the GXXXG motif, the MSD of HIV-1 Env has the critical arginine residue for efficient membrane fusion [46]. The basic amino acid " $\mathrm{K}$ " replaces " $\mathrm{R}$ " in the BIV MSD, but we assume that " $K$ " and "R" have the same function of anchoring the Env glycoprotein in the membrane.

Researchers have predicted two kinds of MSD models, the original model and the "snorkeling" model (West et al., 2001; Yue et al., 2009). The original model requires 25 amino acid residues in the form of an alpha helix. The charged amino acid (R694 of HIV) is buried in the membrane. The "snorkeling" model provides a mechanism by which this relatively short (12 and 15 amino acids in HIV-1 and SIV, respectively) region could be stably accommodated within the membrane. In this model, the membrane-imbedded charged residues (K681 and R694 of HIV; K689 and R705 of SIV) can be neutralized by side-chain interactions with lipid polar head groups. Our study found that the "snorkeling" model, rather than the original model, was suitable for the BIV MSD. This is because the number of amino acids in the BIV MSD is not sufficient to support the original model. Therefore, our results directly support the "snorkeling" model. Our study predicts and verifies the MSD of BIV gp45 for the first time and further clarifies the BIV Env primary structure by confirming the extracellular domain, MSD and cytoplasmic domain of gp45. Finally, based on previous studies regarding Env primary structure (Suarez and Whetstone, 1995; Li et al., 2005) and our results, we constructed the primary structure of BIV Env. The clear fundamental structure of BIV Env will contribute to further studies on BIV Env, such as study of how the ED domain mediates BIV Env fusion and how the CT domain affects the incorporation of Env into the budding viral particle.

There are some limitations to the current study. In the dyetransfer-based assay, the MSD deletion mutation reduced (a)

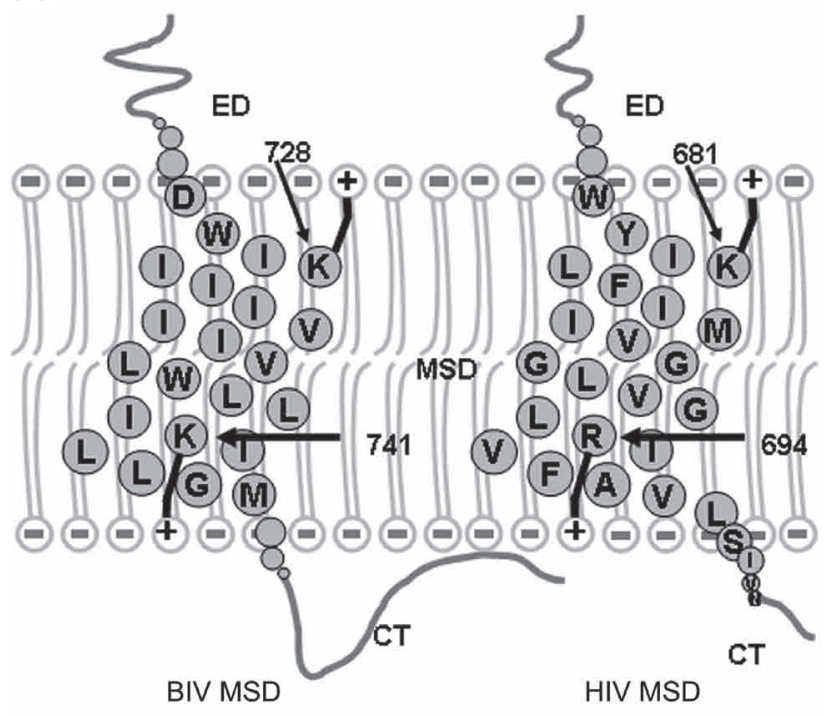

(b)

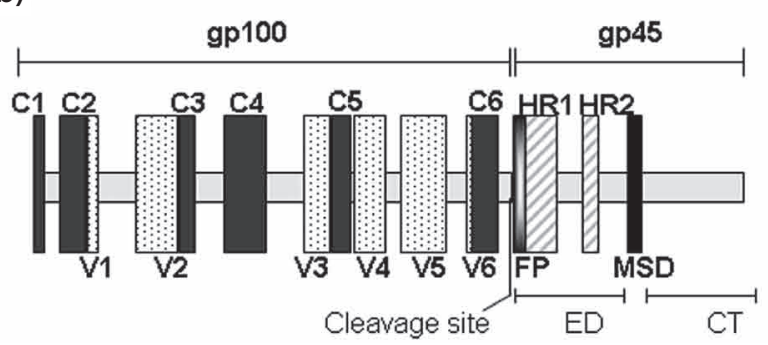

Fig. 5

Schematic structure of the BIV MSD and Env sequence

(a) A "snorkeling" model of the BIV MSD is supported by comparing with the known HIV MSD. In this model, both BIV and HIV MSD have an alpha-helical "core" region, 12 hydrophobic residues between K728/K681 and K741/R694. Interactions of the positively charged side chains of K728/ K681 and K741/R694 (+) with the negatively charged phospholipid head groups (-) are shown. (b) A schematic diagram of the improved primary structure of the BIV Env. In gp100, a total of six conserved regions (c) and six hypervariable regions (V) were defined. The gp45 was divided by MSD into three regions, including ED, MSD and CT. (FP: fusion peptide; HR: heptad repeat; MSD: membrane-spanning domain; ED: extracellular domain; CT: cytoplasmic domain).

cell-cell fusion. To examine how the MSD deletion mutant influences the fusogenicity of the BIV Env, we wanted to establish whether this decrease in fusion resulted from an altered transport and expression on the cell surface using flow cytometry, however the commercial antibody was not available. However, the cell distribution and localization characteristics of BIVgp45 and BIVgp45-nonMSD were consistent. This result excluded the possibility that the absence of the MSD altered the biosynthesis of the glycoprotein precursor or its transport to the Golgi network for comple- 
tion of carbohydrate processing and cleavage into the SU and TM subunits.

In conclusion, we determined that the BIV MSD is located at D170 to M191 amino acids of gp45, and BIV MSD has similar hydrophobic and structural characteristics with other members of the lentivirus family. Moreover, this study improves the understanding of the MSD of lentiviruses. We found that the GXXXG was located mostly at the helix-helix interface of membrane-spanning alpha-helices, but not all of the MSD contained the GXXXG motif, such as the BIV MSD. We also determined that, instead of the original model, the "snorkeling" model represents the BIV MSD structure in the lipid bilayer. In addition, we provided a fundamental structure for further research on the function of Env, such as cell-cell fusion. More in-depth studies of key amino acids and the core area of BIV MSD should be performed in the near future. If BIV Env antibody is available, other viral properties, such as processing, transportation and expression of Env, may also be involved.

Acknowledgments. We are grateful to Dr. Charles Wood of the University of Nebraska Lincoln for support with the plasmid BIV 127 and HIV pNL4-3. We are also grateful to Dr. Wentao Qiao of the Nankai University for providing the plasmid pcBIV. This work was supported by grants from the National Natural Science Foundation of China (81371820, 81571987) and the Ph.D. Candidate Research Innovation Fund of Nankai University (2015) (68150003).

\section{References}

Abrahamyan LG, Markosyan RM, Moore JP, Cohen FS, Melikyan GB (2003): Human immunodeficiency virus type 1 Env with an intersubunit disulfide bond engages coreceptors but requires bond reduction after engagement to induce fusion. J. Virol. 77, 5829-5836. https://doi.org/10.1128/ JVI.77.10.5829-5836.2003

Albernaz TT, Leite RC, Reis JK, de Sousa Rodrigues AP, da Cunha Kassar T, Resende CF, de Oliveira CH, Silva R, Salvarani FM, Barbosa JD (2015): Molecular detection of bovine immunodeficiency virus in water buffalos (Bubalus bubalis) from the Amazon region, Brazil. Trop. Anim. Health Prod. 47, 1625-1628. https://doi.org/10.1007/ s11250-015-0884-6

Bhakta SJ, Shang L, Prince JL, Claiborne DT, Hunter E (2011): Mutagenesis of tyrosine and di-leucine motifs in the HIV-1 envelope cytoplasmic domain results in a loss of Env-mediated fusion and infectivity. Retrovirology 8, 37. https://doi.org/10.1186/1742-4690-8-37

Bhatia S, Patil SS, Sood R (2013): Bovine immunodeficiency virus: a lentiviral infection. Indian. J. Virol. 24, 332-341. https:// doi.org/10.1007/s13337-013-0165-9

Bosch ML, Earl PL, Fargnoli K, Picciafuoco S, Giombini F, WongStaal F, Franchini G (1989): Identification of the fusion peptide of primate immunodeficiency viruses. Science 244, 694-697. https://doi.org/10.1126/science.2541505

Braun MJ, Lahn S, Boyd AL, Kost TA, Nagashima K, Gonda MA (1988): Molecular cloning of biologically active proviruses of bovine immunodeficiency-like virus. Virology $167,515-523$

Chan DC, Fass D, Berger JM, Kim PS (1997): Core structure of gp41 from the HIV envelope glycoprotein. Cell 89, 263-273. https://doi.org/10.1016/S0092-8674(00)80205-6

Cohen FS, Melikyan GB (1998): Methodologies in the study of cellcell fusion. Methods 16, 215-226. https://doi.org/10.1006/ meth.1998.0670

Cosson P, Bonifacino JS (1992): Role of transmembrane domain interactions in the assembly of class II MHC molecules. Science 258, 659-662. https://doi.org/10.1126/science. 1329208

Freed EO, Delwart EL, Buchschacher GL, Jr., Panganiban AT (1992): a mutation in the human immunodeficiency virus type 1 transmembrane glycoprotein gp41 dominantly interferes with fusion and infectivity. Proc. Natl. Acad. Sci. USA 89, 70-74. https://doi.org/10.1073/pnas.89.1.70

Freed EO, Myers DJ, Risser R (1990): Characterization of the fusion domain of the human immunodeficiency virus type 1 envelope glycoprotein gp41. Proc. Natl. Acad. Sci. USA 87, 4650-4654. https://doi.org/10.1073/pnas.87.12.4650

Fujiyoshi Y (1998): The structural study of membrane proteins by electron crystallography. Adv. Biophys. 35, 25-80. https:// doi.org/10.1016/S0065-227X(98)80003-8

Gomez Corredor A, Archambault D (2009): The bovine immunodeficiency virus rev protein: identification of a novel lentiviral bipartite nuclear localization signal harboring an atypical spacer sequence. J. Virol. 83, 12842-12853. https://doi.org/10.1128/JVI.01613-09

Gomez Corredor A, Archambault D (2012): The bovine immunodeficiency virus Rev protein: identification of a novel nuclear import pathway and nuclear export signal among retroviral Rev/Rev-like proteins. J. Virol. 86, 4892-4905. https://doi.org/10.1128/JVI.05132-11

Gonda MA, Braun MJ, Carter SG, Kost TA, Bess JW, Jr., Arthur LO, Van der Maaten MJ (1987): Characterization and molecular cloning of a bovine lentivirus related to human immunodeficiency virus. Nature 330, 388-391. https:// doi.org/10.1038/330388a0

Guo HY, Ma YG, Gai YM, Liang ZB, Ma J, Su Y, Zhang QC, Chen QM, Tan J (2013): Bovine HEXIM1 inhibits bovine immunodeficiency virus replication through regulating BTat-mediated transactivation. Vet. Res. 44, 21. https:// doi.org/10.1186/1297-9716-44-21

Kondo N, Miyauchi K, Meng F, Iwamoto A, Matsuda Z (2010): Conformational changes of the HIV-1 envelope protein during membrane fusion are inhibited by the replacement of its membrane-spanning domain. J. Biol. Chem. 285, 14681-14688. https://doi.org/10.1074/jbc.M109.067090

Li S, Zhu J, Peng Y, Cui S, Wang C, Gao GF, Tien P (2005): Characterization of BIV Env core: implication for mechanism of BIV-mediated cell fusion. Biochem. Biophys. Res. Commun. 329, 603-609. https://doi.org/10.1016/j. bbrc.2005.01.160 
Liu B, Zhao X, Shen W, Kong X (2015): Evidence for the antisense transcription in the proviral R29-127 strain of bovine immunodeficiency virus. Virol. Sin. 30, 224-227. https:// doi.org/10.1007/s12250-015-3559-6

Lu L, Zhu Y, Huang J, Chen X, Yang H, Jiang S, Chen YH (2008): Surface exposure of the HIV-1 env cytoplasmic tail LLP2 domain during the membrane fusion process: interaction with gp41 fusion core. J. Biol. Chem. 283, 16723-16731. https://doi.org/10.1074/jbc.M801083200

Lu M, Blacklow SC, Kim PS (1995): A trimeric structural domain of the HIV-1 transmembrane glycoprotein. Nat. Struct. Biol. 2, 1075-1082. https://doi.org/10.1038/nsb1295-1075

Miyauchi K, Komano J, Yokomaku Y, Sugiura W, Yamamoto N, Matsuda Z (2005): Role of the specific amino acid sequence of the membrane-spanning domain of human immunodeficiency virus type 1 in membrane fusion. J. Virol. 79, 4720-4729. https://doi.org/10.1128/ JVI.79.8.4720-4729.2005

Moller S, Croning MD, Apweiler R (2001): Evaluation of methods for the prediction of membrane spanning regions. Bioinformatics 17, 646-653. https://doi.org/10.1093/ bioinformatics/17.7.646

Munoz-Barroso I, Durell S, Sakaguchi K, Appella E, Blumenthal R (1998): Dilation of the human immunodeficiency virus-1 envelope glycoprotein fusion pore revealed by the inhibitory action of a synthetic peptide from gp41. J. Cell. Biol. 140, 315-323. https://doi.org/10.1083/jcb.140.2.315

Owens RJ, Burke C, Rose JK (1994): Mutations in the membranespanning domain of the human immunodeficiency virus envelope glycoprotein that affect fusion activity. J. Virol. $68,570-574$.

Postler TS, Desrosiers RC (2012): The cytoplasmic domain of the HIV-1 glycoprotein gp41 induces NF-kappaB activation through TGF-beta-activated kinase 1. Cell Host Microbe. 11, 181-193. https://doi.org/10.1016/j. chom.2011.12.005

Raja NU, Vincent MJ, Abdul Jabbar M (1994): Vpu-mediated proteolysis of gp160/CD4 chimeric envelope glycoproteins in the endoplasmic reticulum: requirement of both the anchor and cytoplasmic domains of CD4. Virology 204, 357-366. https://doi.org/10.1006/viro.1994.1540

Reuven EM, Dadon Y, Viard M, Manukovsky N, Blumenthal R, Shai Y (2012): HIV-1 gp41 transmembrane domain interacts with the fusion peptide: implication in lipid mixing and inhibition of virus-cell fusion. Biochemistry 51, 2867-2878. https://doi.org/10.1021/bi201721r

Salzwedel K, West JT, Hunter E (1999): A conserved tryptophanrich motif in the membrane-proximal region of the human immunodeficiency virus type 1 gp41 ectodomain is important for Env-mediated fusion and virus infectivity. J. Virol. 73, 2469-2480.

Senes A, Engel DE, DeGrado WF (2004): Folding of helical membrane proteins: the role of polar, GxxxG-like and proline motifs. Curr. Opin. Struct. Biol. 14, 465-479. https://doi. org/10.1016/j.sbi.2004.07.007
Shang L, Hunter E (2010): Residues in the membrane-spanning domain core modulate conformation and fusogenicity of the HIV-1 envelope glycoprotein. Virology 404, 158-167. https://doi.org/10.1016/j.virol.2010.03.016

Shang L, Yue L, Hunter E (2008): Role of the membrane-spanning domain of human immunodeficiency virus type 1 envelope glycoprotein in cell-cell fusion and virus infection. J. Virol. 82, 5417-5428. https://doi.org/10.1128/JVI.02666-07

Suarez DL, Whetstone CA (1995): Identification of hypervariable and conserved regions in the surface envelope gene in the bovine lentivirus. Virology 212, 728-733. https://doi. org/10.1006/viro.1995.1532

Tan K, Liu J, Wang J, Shen S, Lu M (1997): Atomic structure of a thermostable subdomain of HIV-1 gp41. Proc. Natl. Acad. Sci. USA 94, 12303-12308. https://doi.org/10.1073/ pnas.94.23.12303

Tusnady GE, Simon I (1998): Principles governing amino acid composition of integral membrane proteins: application to topology prediction. J. Mol. Biol. 283, 489-506. https:// doi.org/10.1006/jmbi.1998.2107

Tusnady GE, Simon I (2001): The HMMTOP transmembrane topology prediction server. Bioinformatics 17, 849-850. https://doi.org/10.1093/bioinformatics/17.9.849

Weiss CD (2003): HIV-1 gp41: mediator of fusion and target for inhibition. AIDS Rev. 5, 214-221.

Weissenhorn W, Dessen A, Harrison SC, Skehel JJ, Wiley DC (1997): Atomic structure of the ectodomain from HIV-1 gp41. Nature 387, 426-430. https://doi.org/10.1038/387426a0

Welman M, Lemay G, Cohen EA (2007): Role of envelope processing and gp41 membrane spanning domain in the formation of human immunodeficiency virus type 1 (HIV-1) fusion-competent envelope glycoprotein complex. Virus Res. 124, 103-112. https://doi.org/10.1016/j. virusres.2006.10.009

West JT, Johnston PB, Dubay SR, Hunter E (2001): Mutations within the putative membrane-spanning domain of the simian immunodeficiency virus transmembrane glycoprotein define the minimal requirements for fusion, incorporation, and infectivity. J. Virol. 75, 9601-9612. https://doi. org/10.1128/JVI.75.20.9601-9612.2001

Wilk T, Pfeiffer T, Bukovsky A, Moldenhauer G, Bosch V (1996): Glycoprotein incorporation and HIV-1 infectivity despite exchange of the gp160 membrane-spanning domain. Virology 218, 269-274. https://doi.org/10.1006/ viro. 1996.0190

Yue L, Shang L, Hunter E (2009): Truncation of the membranespanning domain of human immunodeficiency virus type 1 envelope glycoprotein defines elements required for fusion, incorporation, and infectivity. J. Virol. 83, 11588-11598. https://doi.org/10.1128/JVI.00914-09

Zhang W, Wang H, Li Z, Liu X, Liu G, Harris RS, Yu XF (2014): Cellular requirements for bovine immunodeficiency virus Vif-mediated inactivation of bovine APOBEC3 proteins. J. Virol. 88, 12528-12540. https://doi.org/10.1128/ JVI.02072-14 